\title{
Metallic slabs: perturbative treatments based on jellium
}

\author{
C. Fiolhais ${ }^{a, *}$, C. Henriques ${ }^{\text {a,b }}$, I. Sarría ${ }^{\text {c }}$, J.M. Pitarke ${ }^{c, d}$ \\ ${ }^{a}$ Department of Physics, Center for Computational Physics, University of Coimbra, P-3004-516 Coimbra, \\ Portugal \\ ${ }^{\mathrm{b}}$ Departamento de Física, Faculdade de Ciências e Tecnologia, Universidade Nova de Lisboa, \\ P-2825-114 Caparica, Portugal \\ ${ }^{\mathrm{c}}$ Materia Kondentsatuaren Fisika Saila, Zientzi Fakultatea, Euskal Herriko Unibertsitatea, \\ 644 Posta kutxatila, 48080 Bilbo, Basque Country, Spain \\ ${ }^{\mathrm{d}}$ Donostia International Physics Center (DIPC) and Centro Mixto CSIC-UPVIEHU, Donostia, \\ Basque Country, Spain
}

\begin{abstract}
We examine first-order perturbative results based on jellium for the surface energy of slabs of simple metals, using various local pseudo-potentials (Ashcroft, Heine-Abarenkov and evanescent core). The difference between the pseudo-potential and the jellium potential is averaged along the plane parallel to the surface. We compare these perturbative results with those of the stabilized-jellium model (a modification of the regular jellium model in which the perturbation appears in the energy functional right from the outset) and with the output of other perturbative and non-perturbative calculations. (c) 2001 Elsevier Science Ltd. All rights reserved.
\end{abstract}

Keywords: Slabs of simple metals; Local pseudo-potentials; Surface energy

\section{Introduction}

The jellium model is the simplest model which can be used to describe simple metals (metals with $\mathrm{s}$ or $\mathrm{p}$ valence electrons). It avoids the atomic non-uniformities by replacing the ionic cores by a positive uniform background. It describes qualitatively the work function, but it predicts negative surface energies for metals with high valence-electron density.

\footnotetext{
${ }^{*}$ Corresponding author.

E-mail address: tcarlos@teor.fis.uc.pt (C. Fiolhais).
} 
Lang and Kohn [1,2], who were the first to apply the jellium model to surfaces, introduced a perturbative correction to get realistic results. This correction was simple enough to keep most of the original simplicity: the self-consistent density was still that of jellium and the perturbation (difference between the lattice potential and that of the uniform positive background) was averaged over the surface plane. They also improved on the description of the ion-ion interaction through the so-called classic cleavage surface energy. In this way, they obtained face-dependent surface energies, which were always positive.

Later, the influence of the discrete ions in the electronic density was taken into account $[3,4]$ via second-order perturbation theory, which includes the linear response of the electronic distribution to the lattice potential [5]. Rose and Dobson $[3,4]$ were the first to work out second-order surface energy terms, but they used the linear response of bulk jellium, in a kind of local density approximation. Second-order perturbation theory using the linear response of a jellium slab in the random phase approximation has been worked out by Barnett and coworkers [6] and also by Eguiluz [7]. These calculations, which are three-dimensional, show a noticeable influence of the second-order term in the face-dependent surface energies. While the surface energies depend strongly on the exposed face in the firstorder perturbative model of Lang and Kohn, the second-order results show a weaker dependence.

The stabilized-jellium model or structureless pseudo-potential model [8-11], a modification of the regular jellium model in which the perturbation appears in the energy functional right from the outset, includes the perturbation in the effective potential of the self-consistent Kohn-Sham equations and, as a consequence, its effect on the electronic density. Originally, this model was intended to describe flat surfaces and therefore did not include any structure in the averaged perturbation. However, the dependence of the surface energy on the atomic corrugation of a particular face of a metal was incorporated multiplying the flat surface results by a term based on the liquid-drop model $[8,12]$. The results showed a much weaker face dependence than in the work of Lang and Kohn, in agreement with second-order perturbative results. Considering a dipole barrier, due to corrugation, the model was also adapted to improve the previous face-dependent surface energies [13] and to calculate face-dependent work functions $[8,12,13]$.

More elaborated, and therefore more computationally demanding non-perturbative calculations, are now available for surfaces [14,15]. The experimental difficulties to get surface energies of the different surfaces to compare with the perturbative results based on jellium were then obviated by the predictions of these full atomistic calculations. The results of the stabilized-jellium model were found to be fairly realistic for several metals [16].

Some of the above-mentioned calculations were performed for slabs or thin films, i.e., systems made out of a few atomic layers. Slabs, which are convenient to obtain second-order perturbative results for surfaces, are interesting in their own since they exhibit quantum size and self-compression effects. Jellium slabs, showing quantum size effects, were examined in a seminal paper by Schulte [17]. The second-order perturbative energies of Barnett et al. [6] and Eguiluz [7] were obtained for slabs. 
Slabs of stabilized jellium were recently examined by us [18]. We have shown that they are able to describe both quantum-size and self-compression effects.

The above perturbative treatments were implemented using Ashcroft's empty-core pseudo-potential [19] or the local Heine-Abarenkov pseudo-potential [20-22]. In this paper, we study aluminum slabs, with 9 and 17 layers, along the lines of LangKohn's perturbation theory using the Ashcroft, the Heine-Abarenkov, and the recent evanescent core pseudo-potential [23,24], which has the advantage of having a smooth repulsion. We compare our results with those of stabilized jellium and with other perturbative and non-perturbative calculations. Finally, we refer to the possibility of considering stabilized jellium as a zero-order description of a metal surface.

The ultimate goal of our research is to perform systematic first- and second-order perturbative calculations for surfaces of simple metals with the evanescent core pseudo-potential. This will be an extension to surfaces of the systematic treatment of the energetics and mechanical properties we have made for metallic solids in different crystal structures [25]. Although ab initio calculations are nowadays clearly the method of choice for bulk or surface systems, perturbative treatments still have their role to describe trends along the periodic table and along different crystallographic structures. Above all, they can provide understanding of the physics of metal cohesion.

The outline of this paper is as follows. In Section 2, the theoretical background is provided, and in Section 3, the perturbative results are presented. Conclusions appear in Section 4.

\section{Perturbative corrections to jellium model}

\subsection{Electronic and Madelung subsystems}

In order to study a neutral metallic system formed by a fixed lattice of ions and electrons with density $n(\vec{r})$, we start with a superposition of two simple systems: the valence electrons moving in a positive background of density $n_{+}(\vec{r})=\bar{n}$ inside the metal $\left(n_{+}(\vec{r})=0\right.$ outside), where $\bar{n}$ is the mean electronic density, and the ions lattice embedded in a negative background with the same density and size as the positive one.

The first of these subsystems, referred to as the electronic subsystem, is the jellium model. We denote its ground-state energy by $E_{\mathrm{J}}\left[n_{0}\right], n_{0}$ being its electronic density which we assume to be a reasonable approximation to $n(\vec{r})$.

The second subsystem, called Madelung subsystem, has energy $E_{\mathrm{M}}$. If one wishes to correct the jellium description perturbatively, one should consider the interactions within the Madelung system and between this and the electronic subsystem, $E_{\mathrm{M}, \mathrm{e}}$. The total energy is

$$
E=E_{\mathrm{J}}\left[n_{0}\right]+E_{\mathrm{M}}+E_{\mathrm{M}, \mathrm{e}} .
$$

The energy of the jellium model (electronic subsystem) is, in atomic units, given by the following density functional: 


$$
\begin{aligned}
E_{\mathrm{J}}\left[n_{0}\right]= & T\left[n_{0}\right]+E_{\mathrm{xc}}\left[n_{0}\right]+\frac{1}{2} \int \mathrm{d}^{3} r \int \mathrm{d}^{3} r^{\prime} \frac{n_{0}\left(\overrightarrow{r^{\prime}}\right) n_{0}\left(\overrightarrow{r^{\prime}}\right)}{\left|\vec{r}-\overrightarrow{r^{\prime}}\right|} \\
& +\frac{1}{2} \int \mathrm{d}^{3} r \int \mathrm{d}^{3} r^{\prime} \frac{n_{+}\left(\overrightarrow{r^{\prime}}\right) n_{+}(\vec{r})}{\left|\vec{r}-\overrightarrow{r^{\prime}}\right|}-\int \mathrm{d}^{3} r \int \mathrm{d}^{3} r^{\prime} \frac{n_{0}\left(\overrightarrow{r^{\prime}}\right) n_{+}\left(\overrightarrow{r^{\prime}}\right)}{\left|\vec{r}-\overrightarrow{r^{\prime}}\right|},
\end{aligned}
$$

where $T\left[n_{0}\right]$ is the non-interacting kinetic energy, $E_{\mathrm{xc}}\left[n_{0}\right]$ the exchange-correlation energy (often evaluated in the local density approximation, which is incidentally adequate for jellium surfaces [26-28]), and the other terms represent, respectively, the electronic repulsion, the self-repulsion of the positive background and the attraction between the electrons and the positive background.

On the other hand, the Madelung energy is given by

$$
\begin{aligned}
E_{\mathrm{M}}= & \frac{1}{2} \int \mathrm{d}^{3} r \int \mathrm{d}^{3} r^{\prime} \frac{n_{+}(\vec{r}) n_{+}\left(\overrightarrow{r^{\prime}}\right)}{\left|\vec{r}-\overrightarrow{r^{\prime}}\right|}+\frac{1}{2} \sum_{l} \sum_{l^{\prime} \neq l} \frac{Z^{2}}{\left|\vec{R}(l)-\vec{R}\left(l^{\prime}\right)\right|} \\
& -\int \mathrm{d}^{3} r \sum_{l} \frac{Z n_{+}(\vec{r})}{|\vec{R}(l)-\vec{r}|},
\end{aligned}
$$

where the summations run over the ionic positions $\vec{R}(l)$ and $Z$ is the charge of each ion. The first term is the self-repulsion of the negative background, the second the Coulomb repulsion of the ions and the third the interaction between the negative background and the ions.

\subsection{First-order perturbative correction}

In order to simplify the calculations, it is convenient to use a local pseudo-potential, $v_{\mathrm{ps}}$, to represent the ion-electron interactions (from now on, by electrons we mean valence electrons). The pseudo-potential, due to a pseudo-ion of charge $Z$ located at the position $\vec{R}(l)$, can be written as a sum of two contributions: an attractive long-range Coulomb part and a short-range repulsive part:

$$
v_{\mathrm{ps}}(|\vec{r}-\vec{R}(l)|)=-\frac{Z}{|\vec{r}-\vec{R}(l)|}+\omega_{R}(|\vec{r}-\vec{R}(l)|) .
$$

The energy arising from the interactions between the Madelung and the electronic subsystems can be written in the first order as

$$
\begin{aligned}
E_{\mathrm{M}, \mathrm{e}}= & E_{\mathrm{ps}}^{(1)}\left[n_{0}\right]+E_{\mathrm{M},+\mathrm{J}} \\
= & \left\{\int \mathrm{d}^{3} r \sum_{l} n_{0}(\vec{r})\left[v_{\mathrm{ps}}(\vec{r}-\vec{R}(l))\right]+\int \mathrm{d}^{3} r \int \mathrm{d}^{3} r^{\prime} \frac{n_{0}(\vec{r}) n_{+}\left(\overrightarrow{r^{\prime}}\right)}{\left|\vec{r}-\overrightarrow{r^{\prime}}\right|}\right\} \\
& +\left\{\int \mathrm{d}^{3} r \sum_{l} \frac{Z n_{+}(\vec{r})}{|\vec{R}(l)-\vec{r}|}-\int \mathrm{d}^{3} r \int \mathrm{d}^{3} r^{\prime} \frac{n_{+}(\vec{r}) n_{+}\left(\overrightarrow{r^{\prime}}\right)}{\left|\vec{r}-\overrightarrow{r^{\prime}}\right|}\right\} .
\end{aligned}
$$


The terms in the first brackets of (2.5) represent the interaction between the Madelung subsystem and the electrons of the electronic subsystem. They correct the jellium-electron interactions (last term in the right-hand side of (2.2)). The difference $\delta v(\vec{r})$ between the potential of a pseudo-ions lattice and the potential of the jellium background appears in the first-order term, which is the first correction to the jellium model:

$$
E_{\mathrm{ps}}^{(1)}\left[n_{0}\right]=\int \mathrm{d}^{3} r \delta v(\vec{r}) n_{0}(\vec{r})
$$

where

$$
\delta v(\vec{r})=\sum_{l} v_{\mathrm{ps}}(\vec{r}-\vec{R}(l))+\int \mathrm{d}^{3} r^{\prime} \frac{n_{+}\left(\overrightarrow{r^{\prime}}\right)}{\left|\vec{r}-\overrightarrow{r^{\prime}}\right|} .
$$

The second brackets of (2.5) include terms representing the interaction between the Madelung subsystem and the positive background of the electronic subsystem. Taking advantage of the pseudo-potential form (2.4) we may rewrite it as

$$
E_{\mathrm{M},+\mathrm{J}}=-\int \mathrm{d}^{3} r \delta v(\vec{r}) n_{+}(\vec{r})+\int \mathrm{d}^{3} r \sum_{l} \omega_{R}(\vec{r}-\vec{R}(l)) n_{+}(\vec{r}) .
$$

If we add $E_{\mathrm{M}}$ of (2.3) to $E_{\mathrm{M},+\mathrm{J}}$ (as written in the second brackets of (2.5)), we find the second correction to the jellium model, the one which improves the description of the ion-ion interaction:

$$
E_{\mathrm{M}}+E_{\mathrm{M},+\mathrm{J}}=\frac{1}{2} \sum_{l, l^{\prime}, l \neq l^{\prime}} \frac{Z^{2}}{\left|\vec{R}(l)-\vec{R}\left(l^{\prime}\right)\right|}-\frac{1}{2} \int \mathrm{d}^{3} r \int \mathrm{d}^{3} r^{\prime} \frac{n_{+}(\vec{r}) n_{+}\left(\overrightarrow{r^{\prime}}\right)}{\left|\vec{r}-\overrightarrow{r^{\prime}}\right|},
$$

which amounts to replacing the self-repulsion of the background by the repulsion of the point ions.

The described perturbative approach is based on general density functionals and may be applied as well to solids, surfaces, slabs or clusters. Lang and Kohn [1,2] used it to calculate surface properties of metals. Due to translational invariance in the planes parallel to the surface, they took the average of the difference potential $\delta v(\vec{r})$ in (2.6) along the plane parallel to the surface, $\delta v(z)$ ( $z$ being the direction perpendicular to the surface), keeping therefore the one-dimensionality of the underlying jellium model, for which $n_{0}(\vec{r}) \equiv n_{0}(z)$. They wrote

$$
\begin{aligned}
\delta v(z) & =\langle\delta v(\vec{r})\rangle \\
& =\sum_{k} \bar{n} 2 \pi d \int \mathrm{d} x_{\|} v_{\mathrm{ps}}\left(\left[x_{\|}^{2}+\left|z-R_{z}(k)\right|^{2}\right]^{-1 / 2}\right) x_{\|}-\varphi_{+}(z),
\end{aligned}
$$

where $k$ runs over the ionic planes, $d$ denotes the inter-layer spacing, $x_{\|}$is the distance in the plane parallel to the surface from any ion, $R_{z}(k)$ is the position of the $k$ th plane of ions, and $\varphi_{+}(z)$ is the potential due to the uniform positive background. 
Lang and Kohn used the Ashcroft empty-core pseudo-potential [19], which requires for each metal a single parameter, the core radius $r_{C}$ :

$$
v_{\mathrm{ps}}^{A}(|\vec{r}-\vec{R}(l)|)= \begin{cases}0, & |\vec{r}-\vec{R}(l)|<r_{\mathrm{c}}, \\ -Z /|\vec{r}-\vec{R}(l)|, & |\vec{r}-\vec{R}(l)| \geqslant r_{\mathrm{c}} .\end{cases}
$$

A better pseudo-potential is the local form of the Heine-Abarenkov pseudo-potential [20-22], which includes two parameters, $R_{\mathrm{c}}$ and $u$, the first measuring the core radius and the second the amount of repulsion in the core,

$$
v_{\mathrm{ps}}^{H A}(|\vec{r}-\vec{R}(l)|)= \begin{cases}Z u / R_{\mathrm{c}}, & |\vec{r}-\vec{R}(l)|<R_{\mathrm{c}} \\ -Z /|\vec{r}-\vec{R}(l)|, & |\vec{r}-\vec{R}(l)| \geqslant R_{\mathrm{c}} .\end{cases}
$$

A recent pseudo-potential incorporating an exponential decay of the core repulsion, devised for evaluating systematically the energetics of simple metals, is the evanescent core pseudo-potential $[23,24]$, which also depends on two parameters, $R$ and $\alpha$ :

$$
\begin{aligned}
v_{\mathrm{ps}}^{\mathrm{ec}}(|\vec{r}-\vec{R}(l)|)= & -\frac{Z}{|\vec{r}-\vec{R}(l)|}+\frac{Z A}{R} \mathrm{e}^{-\alpha \frac{|\vec{r}-\vec{R}(l)|}{R}} \\
& +\frac{Z}{|\vec{r}-\vec{R}(l)|}\left(1+B \frac{|\vec{r}-\vec{R}(l)|}{R}\right) \mathrm{e}^{-\alpha \frac{\vec{r}-\vec{R}(l) \mid}{R}},
\end{aligned}
$$

where $A$ and $B$ are simple functions of $\alpha$. The smoothness of this potential assures good convergence of its Fourier transform and its suitability to second-order perturbative calculations. It yields overall good results for simple metal solids and clusters [23-25,29,30], when the parameters $R$ and $\alpha$ are fitted to solid-state information.

Perturbative first-order theory can be applied to slabs along the lines of Lang and Kohn using these or other local pseudo-potentials. It can also be done beyond the first order [6,7], but then part of the simplicity of the jellium model is lost.

If one is working with slabs, the surface energy can be extracted from the total slab energy per unit area by subtracting the corresponding bulk energy:

$$
\sigma(L)=\frac{1}{2 A}\left[E(L)-\bar{n} L A \epsilon^{\text {bulk }}\right],
$$

where $A$ is an area, $L$ is the slab width (the width of the jellium background in our system) and $\epsilon^{\text {bulk }}$ is the total energy per particle in the bulk. We can decompose the slab surface energy in various parts. For the functional of (2.1) and considering (2.5), the surface energy reads as

$$
\sigma=\sigma_{\mathrm{J}}+\sigma_{\mathrm{M}}+\sigma_{\mathrm{ps}}^{(1)}+\sigma_{\mathrm{M},+\mathrm{J}} .
$$

The first term in the right-hand side is the jellium surface energy. The second is the Madelung surface energy, for which the following classical cleavage formulae may be used, as Lang and Kohn did [1]:

$$
\sigma_{\mathrm{M}} \approx a Z \bar{n}
$$


where $a$ is a tabulated constant for each face of a given crystal structure. The third term is the first-order pseudo-potential surface energy, which may be calculated using the average value for the perturbative potential given by (2.10),

$$
\sigma_{\mathrm{ps}}^{(1)} \simeq \frac{1}{2} \int_{-\infty}^{\infty} \mathrm{d} z \delta v(z)\left[n(z)-n_{+}(\vec{r})\right] .
$$

Finally, $\sigma_{\mathrm{M},+\mathrm{J}}$ is a cleavage piece which is different from zero only when the ion cores appear out of the jellium surface. We may use, for this term, the expression of Monnier and Perdew [10,11], where the potential is averaged in planes parallel to the surface, as previously done for the difference potential:

$$
\sigma_{\mathrm{M},+\mathrm{J}} \simeq-\int_{\text {Jellium Edge }}^{\infty} \mathrm{d} z\left\langle\sum_{l} \omega_{R}(\vec{r}-\vec{R}(l))\right\rangle \bar{n},
$$

where the angular brackets denote the surface average.

When the width of the slab approaches infinity, the surface energy of the slab approaches the surface energy of the semi-infinite system.

\subsection{Stabilized jellium}

In the stabilized-jellium model, which is based on the perturbative-variational concept of Monnier and Perdew [10,11], the perturbative potential, conveniently averaged, is included in the Kohn-Sham equations. The new self-consistent density is a better approach to the real density than the jellium one. The corrections to the jellium description of the ion-ion and electron-ion interactions are now averaged out: the self-repulsion of the jellium positive background inside each Wigner-Seitz sphere $\tilde{\epsilon}$ is subtracted (the jellium is supposed to describe well the repulsion between cells) and, in the perturbative energy, $\delta v(\vec{r})$ is taken to be constant inside the metal and equal to its average over the volume of the Wigner-Seitz sphere, $\langle\delta v\rangle_{\mathrm{Ws}}$. The stabilized-jellium energy functional is given by

$$
E_{\mathrm{SJ}}[n]=E_{\mathrm{J}}[n]-\tilde{\epsilon} \int \mathrm{d}^{3} r n_{+}(\vec{r})+\langle\delta v\rangle_{\mathrm{WS}} \int \mathrm{d}^{3} r \theta(\vec{r}) n(\vec{r})
$$

or using the equality [8] $\tilde{\epsilon}=\langle\delta v\rangle_{\mathrm{WS}}-e_{\mathrm{M}}-\bar{\omega}_{R}$, where $e_{\mathrm{M}}$ is the (bulk) Madelung energy per particle and $\bar{w}_{R}$ is the pseudo-potential repulsion averaged in the WignerSeitz sphere,

$$
E_{\mathrm{SJ}}[n]=E_{\mathrm{J}}[n]+\left(e_{\mathrm{M}}+\bar{w}_{R}\right) \int \mathrm{d}^{3} r n_{+}(\vec{r})+\langle\delta v\rangle_{\mathrm{WS}} \int \mathrm{d}^{3} r \frac{n_{+}(\vec{r})}{\bar{n}}\left[n(\vec{r})-n_{+}(\vec{r})\right] .
$$

While in the jellium model the energy per particle of the bulk system has a single minimum at a density close to that of sodium

$$
\frac{\mathrm{d} \epsilon_{\mathrm{J}}^{\mathrm{bulk}}}{\mathrm{d} n}=0
$$


in the stabilized-jellium model that energy has a minimum for each metal at the corresponding experimental density

$$
\left.\frac{\mathrm{d} \epsilon_{\mathrm{SJ}}^{\mathrm{bulk}}}{\mathrm{d} n}\right|_{n=n_{\exp }}=0 .
$$

This condition is fulfilled by adjusting a pseudo-potential parameter.

The second term in the right-hand side of (2.19) does not contribute to the surface energy. The stabilized-jellium functional has been recently applied to metal slabs [18], the conclusion being that the surface energy obtained from (2.14) and (2.19)

$$
\sigma_{\mathrm{SJ}}=\sigma_{\mathrm{SJ}, \mathrm{J}}+\sigma_{\mathrm{SJ}, \mathrm{ps}}
$$

gives a reasonable description of aluminum slabs in comparison with ab initio results, but it fails for lithium slabs, a case where the necessity for a non-local pseudopotential is known and for which first-principles calculations showed untypical features for a simple metal [31]. Note that the first term of (2.20) is similar to the jellium surface energy but is evaluated with the self-consistent density obtained from functional (2.19). On the other hand, the second term differs from the Lang-Kohn perturbative term by the use of a three-dimensional average for the perturbative potential instead of a two-dimensional one and by the use of the self-consistent stabilized-jellium electron density instead of the jellium electron density. In summary, though inspired by it, the stabilized-jellium model is not perturbation theory.

In [18], we have shown that the application of the stabilized-jellium model to slabs leads to quantum size effects, i.e., fluctuations in the surface energy and work function, which are similar to those known for the jellium model but are around more realistic values. Moreover, we have shown that, fixing the width of the slab, energy minimization with respect to background variation leads to a higher background density inside the slab, i.e., the system tends to self-compress.

Using functional (2.19) no difference shows up between different crystallographic faces. However, ad hoc modifications of stabilized jellium have been proposed to describe the difference between various exposed faces $[8,12,13]$. In these approaches, the self-consistent density is obtained by considering a face-dependent potential but, for the sake of realism, the use of the latter is avoided in the final evaluation of the first-order surface energy. This methodology describes reasonably well the face-dependence found by more sophisticated methods.

A modification of stabilized jellium has been made by Montag et al. [32], who tried to incorporate the cleavage energy in a phenomenological way (by fitting to empirical surface energies).

\section{Results}

We have considered jellium slabs corresponding to 7 and 19 layers of aluminum (fcc lattice), cut along the three main planes (1 111$),\left(\begin{array}{lll}1 & 0 & 0\end{array}\right)$ and $\left(\begin{array}{lll}1 & 1 & 0\end{array}\right)$ (by decreasing order of planar density and, therefore, by increasing order of interplanar distance). 
We expect perturbation theory based on jellium to converge better for the planes which are most close-packed and therefore more similar to a flat surface.

Fig. 1 represents our three pseudo-potentials for aluminum. The following values for the pseudo-potentials parameters have been used (all in atomic units, except $\alpha$, which is dimensionless): $r_{\mathrm{c}}=1.12$ (Ashcroft); $R_{\mathrm{c}}=1.4017$ and $u=-0.3921$ (HeineAbarenkov); and $R=0.317, \alpha=3.512$ (evanescent core). The first value is simply derived from a stability condition for the bulk energy within first-order perturbation theory. The second pair of values come from a stability condition for the bulk energy at the experimental density and from matching the bulk modulus (within secondorder perturbation theory) to the experimental value [7]. The last pair of values arise again from a stability condition for the bulk energy within second-order and from matching first-order values [23,24] to all-electron values for the number of electrons in the interstitial region (zone between the Wigner-Seitz cell and the inscribed sphere). It has been shown that the latter requirement does not differ much from the demand for a realistic bulk modulus [25].

Jellium surface energies $\sigma_{\mathrm{J}}$ are straightforward to evaluate. The cleavage energy $\sigma_{\mathrm{M}}$ has been taken from $[10,11]$. We note that the differences between the values of $\sigma_{\mathrm{M}}$ for various faces are large. The term $\sigma_{\mathrm{M},+\mathrm{J}}$ is small: it may be even zero depending on the size of the core radius $[10,11]$. The perturbative potentials $\delta v(z)$ corresponding to the different pseudo-potentials are represented in Fig. 2. They enter in the calculation of $\sigma_{\mathrm{ps}}^{(1)}$. We note the better smoothness of the $\delta v(z)$ arising from the evanescent core potential.

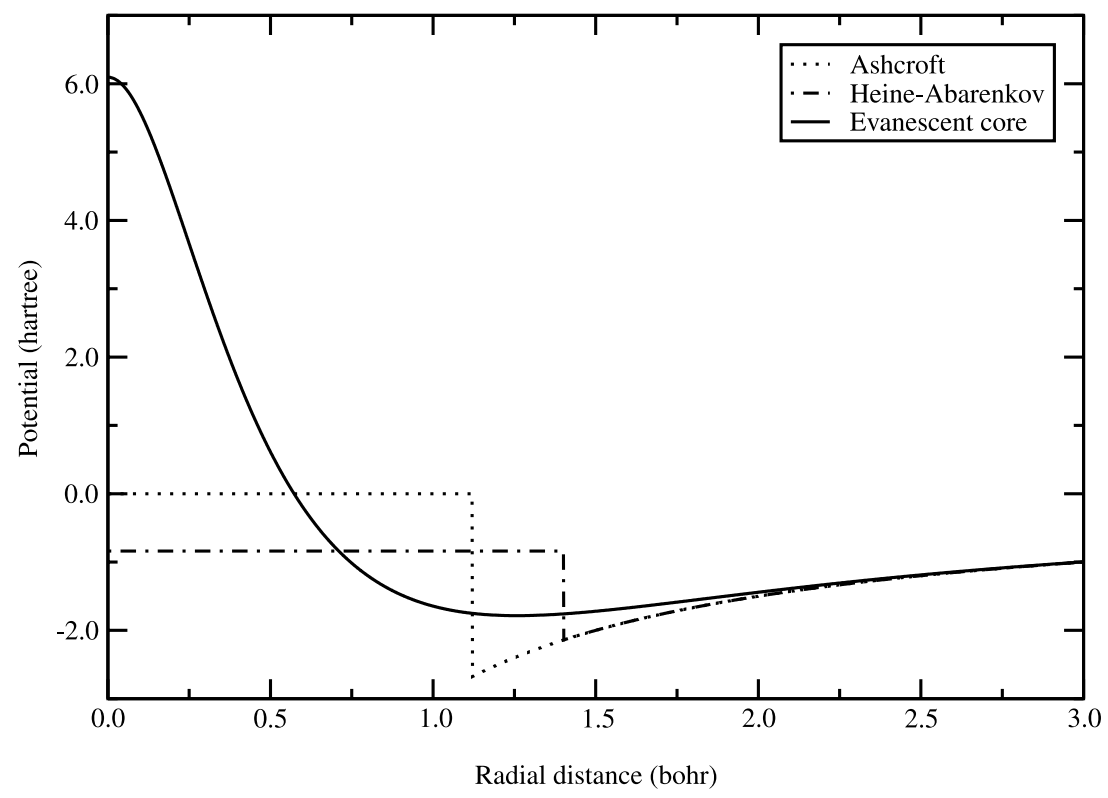

Fig. 1. Three pseudo-potentials for aluminum used in this work as function of radial distance. 


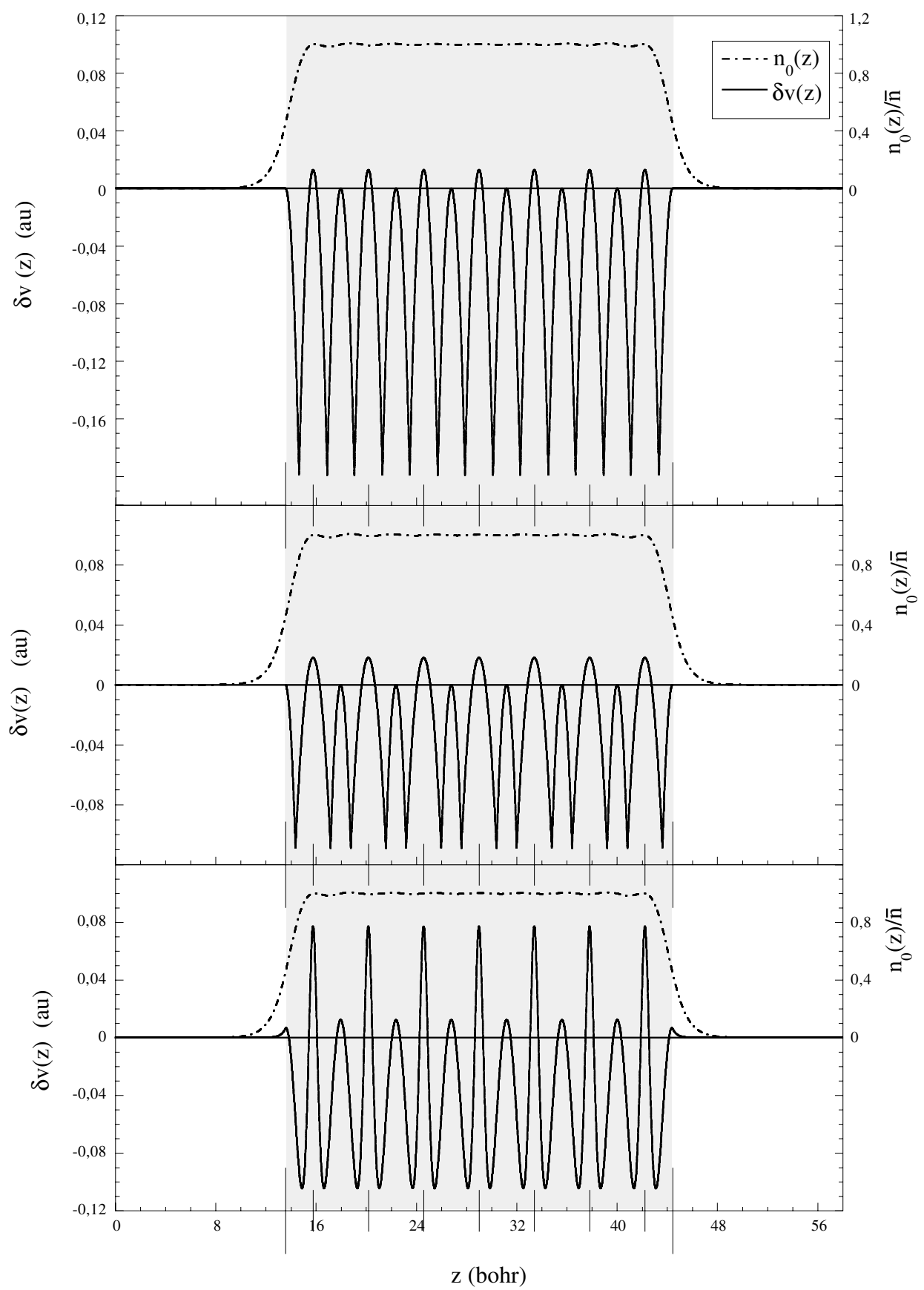

Fig. 2. Jellium electronic density $n_{0}(z)$ and perturbative potential $\delta v(z)$ contributing to first-order energy of slab with 7 layers (( $\left(\begin{array}{lll}1 & 1 & 1\end{array}\right)$ face). Three pictures refer, from top to bottom, to Ashcroft, Heine-Abarenkov and evanescent core pseudo-potentials. The shaded area indicates the background jellium. 
Table 1

First-order surface energies for slabs with 7 and 19 layers of aluminum cut along, respectively, the (1 111$)$, (1 00$)$ and the (1 10$)$ planes ${ }^{\mathrm{a}}$

\begin{tabular}{|c|c|c|c|c|c|c|c|c|c|}
\hline \multirow[t]{2}{*}{ Face } & \multicolumn{2}{|l|}{$\sigma_{\mathrm{J}}$} & \multirow[t]{2}{*}{$\sigma_{\mathrm{M}}$} & \multirow[t]{2}{*}{ Pseudo-potential } & \multicolumn{2}{|l|}{$\sigma_{\mathrm{ps}}^{(1)}$} & \multirow[t]{2}{*}{$\sigma_{\mathrm{M},+\mathrm{J}}$} & \multicolumn{2}{|l|}{$\sigma$} \\
\hline & 7 layers & 19 layers & & & 7 layers & 19 layers & & 7 layers & 19 layers \\
\hline \multirow[t]{3}{*}{$\mathrm{Al}\left(\begin{array}{lll}1 & 1 & 1\end{array}\right)$} & -610.4 & -602.7 & 408.6 & Ashcroft & 1050.5 & 1053.5 & 0 & 851.7 & 856.4 \\
\hline & -610.4 & -602.7 & 408.6 & Heine-Abarenkov & 680.9 & 677.8 & 0 & 479.1 & 483.7 \\
\hline & -610.4 & -602.7 & 408.6 & Evanescent core & 544.9 & 543.5 & -92.7 & 250.4 & 256.7 \\
\hline \multirow[t]{2}{*}{$\operatorname{Al}\left(\begin{array}{lll}1 & 0 & 0\end{array}\right)$} & -613.8 & -603.4 & 1802.8 & Ashcroft & 405.3 & 410.8 & 0 & 1594.3 & 1610.2 \\
\hline & -613.8 & -603.4 & 1802.8 & Heine-Abarenkov & -108.3 & -107.7 & 0 & 1080.7 & 1091.6 \\
\hline \multirow[t]{3}{*}{$\operatorname{Al}\left(\begin{array}{lll}1 & 1 & 0\end{array}\right)$} & -585.6 & -608.3 & 5540.3 & Ashcroft & -1588.6 & -1581.7 & 0 & 3366.1 & 3350.3 \\
\hline & -585.6 & -608.3 & 5540.3 & Heine-Abarenkov & -2315.2 & -2306.2 & -15.4 & 2624.1 & 2610.4 \\
\hline & -585.6 & -608.3 & 5540.3 & Evanescent core & -1986.8 & -1978.1 & -588.7 & 2379.2 & 2365.1 \\
\hline
\end{tabular}

${ }^{\mathrm{a}} \sigma_{\mathrm{J}}, \sigma_{\mathrm{M}}, \sigma_{\mathrm{ps}}^{(1)}$, and $\sigma_{\mathrm{M},+\mathrm{J}}$ are the terms of the surface energy, $\sigma$, following (2.15). The Perdew-Wang [33] correlation functional was used in the jellium term. The other terms were calculated using the approximations expressed by (2.16)-(2.18). All values are in $\mathrm{erg} / \mathrm{cm}^{2}$. 
Table 2

Flat stabilized-jellium surface energies (no corrugation included in the calculations) for slabs with thicknesses corresponding to 7 and 19 layers ${ }^{\mathrm{a}}$

\begin{tabular}{|c|c|c|c|}
\hline Thicknesss & $\sigma_{\mathrm{S}, \mathrm{J}}$ & $\sigma_{\mathrm{SJ}, \mathrm{ps}}$ & $\sigma_{\mathrm{SJ}}$ \\
\hline 7 layers of $\mathrm{Al}\left(\begin{array}{lll}1 & 1 & 1\end{array}\right)$ & -447.9 & 1369.8 & 921.9 \\
\hline 19 layers of $\mathrm{Al}\left(\begin{array}{lll}1 & 1 & 1\end{array}\right)$ & -450.6 & 1376.0 & 925.4 \\
\hline 7 layers of $\mathrm{Al}\left(\begin{array}{lll}1 & 0 & 0\end{array}\right)$ & -445.9 & 1363.2 & 917.3 \\
\hline 19 layers of $\mathrm{Al}(100)$ & -450.2 & 1376.2 & 926.0 \\
\hline 7 layers of $\mathrm{Al}\left(\begin{array}{lll}1 & 10\end{array}\right)$ & -460.1 & 1405.1 & 945.0 \\
\hline 19 layers of $\mathrm{Al}\left(\begin{array}{lll}1 & 10\end{array}\right)$ & -451.5 & 1370.8 & 919.3 \\
\hline
\end{tabular}

${ }^{\mathrm{a}} \sigma_{\mathrm{SJ}, \mathrm{J}}$ and $\sigma_{\mathrm{SJ}, \mathrm{ps}}$ are the terms of the stabilized-jellium surface energy, $\sigma_{\mathrm{SJ}}$, following (2.20). The PerdewWang [33] correlation functional was used in the jellium term. All values are in erg $/ \mathrm{cm}^{2}$.

Table 1 shows total surface energies, together with their components, obtained with the three pseudo-potentials for the slabs with 7 and 19 layers. Table 1 illustrates the importance of the positive Madelung contribution. For the same pseudo-potential the surface energy increases when going from the (1 111$)$ face to the $\left(\begin{array}{lll}1 & 1 & 0\end{array}\right)$ face. The results show a strong dependence on the pseudo-potential, with the Ashcroft result, which in principle is the most unrealistic, being discrepant from the other two (it is always bigger). Without the Madelung term, the perturbative correction of Lang and Kohn would lead to a positive surface energy only for the (1 111 ) face, precisely that considered by those authors.

Table 2 shows the flat stabilized jellium results for aluminum slabs with thicknesses corresponding to 7 and 19 layers. The total surface energies are in general very different not only from the jellium ones but also from first-order perturbation results.

In spite of the quantum size oscillations, slabs may be used to estimate the surface energy of the semi-infinite system. Indeed, the slab with thickness corresponding to 19 layers is a good approximation to the latter. Table 3 allows us to compare our previous slab results with semi-infinite results (Lang-Kohn, stabilized jellium, and other perturbative and non-perturbative results). Actually, the non-perturbative result was obtained with the plane-wave pseudo-potential method for 12 layers separated by a vacuum of 6 layers, but this should represent well the semi-infinite

Table 3

Surface energies obtained by first-order perturbative theory (Lang-Kohn approach [1] with the Ashcroft pseudo-potential), in the second column, and by other methods ${ }^{\mathrm{a}}$

\begin{tabular}{|c|c|c|c|c|c|c|}
\hline Surface & $\sigma$ & $\sigma_{\mathrm{SJ}}^{\text {flat }}$ & $\sigma_{\mathrm{SJ}}^{\text {face }}$ & $\sigma_{\mathrm{MP}}$ & $\sigma_{\mathrm{RD}}$ & $\sigma_{\mathrm{SBH}}$ \\
\hline $\mathrm{Al}\left(\begin{array}{lll}1 & 1 & 1\end{array}\right)$ & 842 & 925 & 938 & 643 & 1065 & 939 \\
\hline $\mathrm{Al}\left(\begin{array}{lll}1 & 0 & 0\end{array}\right)$ & 1631 & 925 & 1087 & 1460 & 1160 & 1081 \\
\hline $\mathrm{Al}\left(\begin{array}{lll}1 & 1 & 0\end{array}\right)$ & 3393 & 925 & 1679 & 2870 & 1700 & 1090 \\
\hline
\end{tabular}

${ }^{\mathrm{a}}$ The third column refers to flat stabilized jellium [8], $\sigma_{\mathrm{SJ}}^{\text {flat }}$, the fourth to face-dependent stabilized jellium [13], $\sigma_{\mathrm{SJ}}^{\text {face }}$, the fifth to the perturbative-variational method of Monnier and Perdew [10], $\sigma_{\mathrm{MP}}$, the sixth to the second-order perturbative theory of Rose and Dobson [3], $\sigma_{\mathrm{RD}}$, and the seventh to Schöchlin et al. [34] first-principles non-perturbative results, $\sigma_{\mathrm{SBH}}$. In these works, the semi-infinite system is considered except in the last one, where a slab with 12 layers was taken. All calculations were done in the local density approximation. All values are in $\mathrm{erg} / \mathrm{cm}^{2}$. 
system. Accepting this non-perturbative as the best result, we have to conclude that its agreement with the (1 111 ) surface energy of Lang and Kohn, who used the Aschroft potential, is accidental. The same pseudo-potential for any other face leads to disagreement. However, the most striking conclusion of Table 3 is that the facedependent stabilized-jellium model can emulate quite well the second-order perturbative result of Rose and Dobson and also the non-perturbative calculation, with the single exception of the (1 110$)$ case.

\section{Conclusions}

We have studied within perturbation theory surface energies of metallic slabs taking as zero-order the jellium model. Our results allow us to conclude that firstorder surface energies depend strongly on the pseudo-potential used. For aluminum slabs, the Heine-Abarenkov and the evanescent core potentials give similar results, while the Ashcroft potential differs from those two (it differs more for the least dense surfaces). It should therefore be used with some caution. On the other hand, the firstorder perturbation turns out to be too large for the least dense surfaces, being imperative to correct it through second-order terms. We are implementing secondorder perturbative calculations using the slab response function. We pointed out the importance of the Madelung energy, which should therefore be evaluated as far as possible without any approximations.

We stressed the usefulness of the stabilized-jellium model, which keeps the essential simplicity of jellium, while reproducing various first principles results for slabs and surfaces. As an extension of that model, we may take as zero's order the stabilized-jellium energy instead of the jellium one (since the perturbation is strong for all aluminum faces, it should, after all, be a better starting point for perturbation theory) and to take as first-order perturbation, the difference between the pseudopotential of stabilized jellium and an adequate pseudo-potential. Work along these lines is in progress.

\section{Acknowledgements}

The authors gratefully acknowledge J.P. Perdew (Tulane University, USA) for useful discussions. They also acknowledge F. Nogueira (Coimbra University, Portugal) for providing some bulk results and for helping in preparing the manuscript. This project has been supported by the Portuguese PRAXIS XXI Program (Project PRAXIS/2/2.1/FIS/473/94) and by the University of the Basque Country, the Basque Hezkuntza, Unibertsitate eta Ikerketa Saila, and the Spanish Ministerio de Educación y Cultura. 


\section{References}

[1] N.D. Lang, W. Kohn, Phys. Rev. B 1 (1970) 4555.

[2] N.D. Lang, W. Kohn, Phys. Rev. B 3 (1971) 1215.

[3] J.H. Rose, J.F. Dobson, Solid State Comm. 37 (1981) 91.

[4] J.F. Dobson, J.H. Rose, J. Phys. C 15 (1982) 7429.

[5] J.F. Dobson, G.H. Harris, Phys. Rev. B 27 (1983) 6542.

[6] R.N. Barnett, R.G. Barrera, C.L. Cleveland, U. Landman, Phys. Rev. B 28 (1983) 1667,1685.

[7] A.G. Eguiluz, Phys. Rev. B 35 (1987) 5473.

[8] J.P. Perdew, H.Q. Tran, E.D. Smith, Phys. Rev. B 42 (1990) 11627.

[9] C. Fiolhais, J.P. Perdew, Phys. Rev. B 45 (1992) 6207.

[10] R. Monnier, J.P. Perdew, Phys. Rev. B 17 (1978) 2595.

[11] R. Monnier, J.P. Perdew, Phys. Rev. B 22 (1980) 1134(E).

[12] J.P. Perdew, Prog. Surf. Sci. 48 (1995) 245.

[13] A. Kiejna, Phys. Rev. B 47 (1993) 7361.

[14] H.L. Skriver, N.M. Rosengaard, Phys. Rev. B 43 (1991) 9538

[15] H.L. Skriver, N.M. Rosengaard, Phys. Rev. B 46 (1992) 7157.

[16] J.E. Inglesfield, in: F.R. De Boer, D.Gs. Pettifor (Eds.), Cohesion and Structure of Surfaces, vol. 4, Elsevier, Amsterdam, 1995.

[17] F.K. Schulte, Surf. Sci. 55 (1976) 427.

[18] I. Sarria, C. Henriques, C. Fiolhais, J.M. Pitarke, Phys. Rev. B 62 (2000) 1699.

[19] N.W. Ashcroft, Phys. Lett. 23 (1966) 48.

[20] V. Heine, D. Weaire, in: H. Ehrenreich, F. Seitz, D. Turnbull (Eds.), Solid State Physics, vol. 24, Academic Press, New York, 1970.

[21] I. Abarenkov, V. Heine, Philos. Mag. 12 (1965) 529.

[22] V. Heine, I. Abarenkov, Philos. Mag. 9 (1964) 451.

[23] C. Fiolhais, J.P. Perdew, S.Q. Armster, J.M. MacLaren, M. Brajczewska, Phys. Rev. B 51 (1995) 14001.

[24] C. Fiolhais, J.P. Perdew, S.Q. Armster, J.M. MacLaren, M. Brajczewska, Phys. Rev. B 53 (1996) 13193(E).

[25] F. Nogueira, C. Fiolhais, J.P. Perdew, Phys. Rev. B 59 (1999) 2570.

[26] J.M. Pitarke, A. Eguiluz, Phys. Rev. B 57 (1998) 6329.

[27] S. Kurth, J.P. Perdew, Phys. Rev. B 59 (1999) 10461.

[28] Z. Yan, J.P. Perdew, S. Kurth, C. Fiolhais, L. Almeida, Phys. Rev. B 61 (2000) 2595.

[29] F. Nogueira, C. Fiolhais, J. He, J.P. Perdew, A. Rubio, J. Phys: Cond. Matt. 8 (1996) 287.

[30] C. Fiolhais, F. Nogueira, C. Henriques, Prog. Surf. Sci. 53 (1996) 315.

[31] U. Birkenheur, J.C. Boettger, N. Rosch, Surf. Sci. 341 (1995) 103.

[32] B. Montag, P.-G. Reinhard, J. Meyer, Z. Phys. D 32 (1994) 125.

[33] J.P. Perdew, Y. Wang, Phys. Rev. B 45 (1992) 13244.

[34] J. Schöchlin, K.P. Bohnen, K.M. Ho, Surf. Sci. 324 (1995) 113. 